hep-th/0411271

SLAC-PUB-10859

SU-ITP-4/41

\title{
A New Handle on de Sitter Compactifications
}

\begin{abstract}
Alex Saltman and Eva Silverstein *
We construct a large new class of de Sitter (and anti de Sitter) vacua of critical string theory from flux compactifications on products of Riemann surfaces. In the construction, the leading effects stabilizing the moduli are perturbative. We show that these effects self-consistently dominate over standard estimates for further $\alpha^{\prime}$ and quantum corrections, via tuning available from large flux and brane quantum numbers.
\end{abstract}

November 2004

* SLAC and Department of Physics, Stanford University, Stanford, CA 94309 


\section{Introduction}

The construction and study of string theory models of de Sitter space is of interest for many reasons. It provides a basis for phenomenological models of dark energy and inflation from string theory and also provides a concrete microphysical framework in which to investigate holography in the cosmological context. The emerging variety of discrete, physically connected solutions has interesting implications for the global structure of the universe according to string theory and challenges conventional naturalness assumptions in the resulting particle phenomenology.

In this paper, we present a new class of compactifications of string theory, based on Riemann surfaces, yielding de Sitter (as well as anti de Sitter) solutions in the resulting four-dimensional effective field theory. There are many potential compactifications of string theory beyond those which classically preserve a massless gravitino; those we consider here form a particularly simple illustrative set of examples. They realize the case of KaluzaKlein scale breaking of supergravity, complementing the previous classes of models with sub-KK scale supersymmetry breaking [1] and string scale supersymmetry breaking [2]. This class turns out to be particularly simple, involving basic aspects of the geometry of Riemann surfaces while generating sufficiently generic contributions to the moduli potential to meta-stabilize the system perturbatively.

Although generic vacua of this class will have high-scale supersymmetry breaking, lowenergy supersymmetric particle physics models may be included, as the communication of the supersymmetry breaking in the gravity sector allows for a separation of scales between the matter superpartner masses and the KK-scale gravitino masses. In any case, the apparent proliferation of vacua in this new class suggests that (as would be expected from genericity) their number may significantly exceed that of the more symmetric choices of vacua. We should also emphasize that this class is itself likely to be a small corner of the space of possibilities.

Our construction starts from a compactification on a Riemann surface of genus at least two whose complex-structure moduli we stabilize via fluxes in a simple manner (explicitly for genus 2 and 3). Reducing from the critical dimension (for simplicity) this leaves four remaining compact dimensions which can be compactified in many ways. Perhaps the simplest option, which we exercise, is to consider these to also be Riemann surfaces, giving us a compactification on the product of three Riemann surfaces.

The low-energy theory obtained from a flux compactification on Riemann surfaces

alone has remaining tadpoles for the dilaton and the volume of each surface. In order to 
stabilize these moduli we add 7-branes, described locally via an F-theory compactification on an elliptically fibered fourfold. We argue that, by a generalization of a similar mechanism used in [3,1], the set of intersecting wrapped 7-branes (equivalently the topology of the F-theory fourfold) provides an anomalous negative 3-brane charge and tension and therefore a tunable negative contribution to the potential. More specifically, in order to obtain a net negative contribution to this part of the moduli potential and the three-form fluxes, while satisfying the constraints of Gauss' law in the compactification, we need to includes sets of 7-branes separated from sets of anti 7-branes, which have a similar anomalous negative 3-brane tension (without net charge), and behave in our stabilized regime as a generalization of combinations of $\mathrm{O} 3$ planes and anti O3-planes. This procedure retains the general property of KK scale breaking of supergravity, while providing needed forces to stabilize the volumes and dilaton perturbatively.

We will at various points make genericity arguments, for example regarding the positions that the 7-branes settle and regarding a technical simplification of the axion contribution to the dynamics. These arguments are based on contributions such as ambient fluxes generic to our construction but we will not exhibit their effects in detail (for the 7-brane moduli, our treatment is thus similar to [3,1]). In any case, we will provide an explicit perturbative stabilization mechanism for the dilaton and volume moduli, as well as Riemann-surface complex-structure moduli.

\subsection{Relations to previous works}

The previous string-theoretic models of de Sitter fall into two classes-those based on supersymmetric low-energy effective theories arising from Calabi-Yau compactifications of critical string theory [1], and those based on string-scale supersymmetry breaking in supercritical limits of string theory [2]. The present models lie in between these classes. Here supersymmetry is generically broken in the gravity sector at an intermediate scale corresponding to the Kaluza-Klein scale of the compactification. This may still allow for low-energy supersymmetry in the matter sector, but with intermediate scale gravitini国;

1 Many important works have appeared recently in the area of moduli stabilization and the discretuum, including for example [4, 5, 6, 7, 8,9, 10, 11, 12, 13, 14, 15, 16, 17, 18, 19, 20, 21, 22, 23, 24, 25, 26, 27 $28,29,30,31,32,33,34$. The works for example [35, 36] considered compactification on hyperbolic spaces, and the work [37] considered compactifications of field theories on Riemann surfaces.

2 This may be of use in addressing the gravitino problem, as discussed recently in [38] [39] and references therein. 
more generally these models have non-supersymmetric spectra at low energies (and some of them may fit into the framework [7]). The complex-structure moduli of the Riemann surface are stabilized by one-form fluxes on pairs of dual one-cycles in a way similar to the stabilization of complex-structure moduli of the Calabi-Yau in [3] by three-form fluxes on pairs of dual three-cycles. The dilaton tadpole present at leading order in the expansion in string coupling is of the same form as that arising in supercritical limits of perturbative string theory [2]. Like the models [2], Riemann-surface compactifications exhibit sufficient forces to stabilize all moduli perturbatively, while like the models [1], the species enhancement to the effective couplings is manifestly controllable.

\subsection{A note on control.}

For readers most familiar with low-energy-supersymmetric compactifications, it is worth reviewing the methods for theoretical control that exist in the absence of supersymmetry below the Kaluza-Klein scale. We use a controlled perturbation expansion in the low-energy effective field theory derived from string theory. This is obtained by introducing by hand sufficiently large flux and brane quantum numbers to ensure that couplings are stabilized at small enough values and volumes at large enough values that the solution has small flux energy density and small effective couplings (including enhancements from numbers of species running in loops). This procedure, and the control it affords, does not depend on low-energy supersymmetry.

As in flux compactifications on spheres (familiar in recent years for their role in the AdS/CFT correspondence), the tadpoles arising from the curvature in the geometry are cancelled by forces introduced by other ingredients arising at higher orders in the expansion in inverse volumes and string coupling (such as fluxes, wrapped branes and orientifolds). It is not necessary for consistency to cancel tadpoles order by order in string perturbation theory-indeed such a procedure would guarantee that the dilaton is not fixed perturbatively.

3 Indeed, low-energy supersymmetric non-renormalization theorems, if too powerful, can preclude the generation of sufficient forces to fix moduli perturbatively. Thus they can require the tuning of classical effects against non-perturbative effects, a procedure that turns out to be possible (and elegant) [1] but is arguably harder than the tuning needed to play different orders of perturbation theory off of each other. Conversely, with either $\mathcal{N}=1$ supersymmetry or $\mathcal{N}=0$, the moduli potential suffers from quantum corrections at arbitrary loop orders, and hence perturbative control must be established in much the same way in both cases. 


\section{Riemann surface flux compactification}

Consider string theory compactified on a Riemann surface $\Sigma$ of genus $h$. The light degrees of freedom (which we will refer to as "moduli") arising from the metric on the Riemann surface are as follows. Using diffeomorphism invariance, we can reduce the metric degrees of freedom to the complex structure moduli, which are zero modes, the conformal factor, whose overall volume mode we will keep and whose higher KK modes we will selfconsistently ignore, and off-diagonal metric modes, which we will ignore because they are massive due to the absence of continuous isometries in higher-genus Riemann surfaces.

As an example, one can consider a configuration in which the metric has constant curvature on the Riemann surface-this is the configuration toward which the system evolves in the absence of other sources, or for sources which are uniformly distributed on the surface. For $h \geq 2$ the surface has $3 h-3$ complex-structure moduli which correspond to changes of metric that do not affect the curvature, and hence are massless deformations about that configuration. In addition, there is a scalar field corresponding to the volume of the surface, arising from the lowest mode of the conformal factor of the metric.

We will introduce fluxes and other ingredients to stabilize these moduli as well as the other potentially runaway moduli (such as the dilaton) arising in a full compactification down to four dimensions. In particular, we will obtain solutions with a local minimum of the potential energy above zero, i.e. metastable de Sitter solutions. Because the KaluzaKlein modes start with large masses from their internal gradients, we will ignore their dynamics here; in our final analysis we will see that the KK scale can be tuned to be parametrically higher than the curvature scale of the four-dimensional spacetime, and that the masses for the moduli can be arranged to be parameterically lighter than the KK masses in the minimum if so desired.

The Einstein term $\sqrt{g} \mathcal{R}$ integrated over the Riemann surface produces a tree-level contribution to the low-energy effective potential proportional to $2 h-2$. In four-dimensional Einstein frame (appropriate after further compactification on a space $X$ of volume $V_{X}$ in string units) this contribution scales liket

$$
U_{\mathcal{R}} \sim \frac{1}{l_{4}^{4}}(2 h-2) \frac{g_{s}^{2}}{V_{\Sigma}^{2} V_{X}}
$$

4 See [40] for a basic review of the potential energy arising from various ingredients in string compactifications. 
where $l_{4}$ is the four-dimensional Planck length. The contribution (2.1) provides a treelevel force on a combination of the dilaton and volume moduli, but does not depend on the $3 h-3$ (for $h \geq 2$ ) complex moduli of the Riemann surface.

More generally, we will consider 7 -branes embedded in $\Sigma$, described locally via Ftheory on an appropriate fourfold geometry. These contribute positive potential energy from their tension in addition to negative contributions arising from F-theoretic curvature couplings which we will review later.

In standard F-theory constructions, one considers F-theory compactified on an elliptically-fibered Calabi-Yau manifold, which in the corresponding type IIB description is a set of intersecting 7-branes embedded in the base of the fibration [41. The positive tension contributions of the 7-branes add to the tree level tadpole in (2.1). For example, the type IIB description of F-theory on K3 amounts to 24 (p,q) 7-branes embedded in a genus $h=0$ compactification, in such a way that the 7-branes cancel the term (2.1) completely. More generally, if we view a higher-genus Riemann surface $\Sigma$ as a $\mathbb{P}^{1}$ with handles attached, we can for example consider the same set of 7 -branes on $\Sigma$. In the F-theory description, we are patching into the base of the fibration a trivial fibration of a $T^{2}$ over a set of handles. Allowing for multiple such sets of 24 (including separated antibrane sets in general), this means that (2.1) becomes

$$
U_{\mathcal{R}, 7 B s} \sim \frac{1}{l_{4}^{4}}\left(2 h+2\left(n_{7}-1\right)\right) \frac{g_{s}^{2}}{V_{\Sigma}^{2} V_{X}}
$$

where $n_{7}$ represents the number of sets of 24 -branes (and/or anti 7-branes) included in the surface. Here we used that the $h=0$ contribution in (2.1) is cancelled by the effects of one such set of sevenbranes; this is a good approximation at large volume, where the SUSY breaking scale is much smaller than the 7-brane tensions. More generally, we can consider additional D7-branes and anti-D7-branes; their effects on the potential energy will arise at the next order in $g_{s}$. In general, we will find the need for sets of both 7 -branes and anti 7-branes; we will discuss their position moduli in $\S 3$.

Fluxes threading one-cycles of $\Sigma$ will prove useful for stabilizing the volume and dilaton, and also yield classical forces on the complex-structure moduli from the flux kinetic terms $\mathcal{L}_{\text {flux }} \sim-\int F \wedge * F$; we will study this explicitly in the next subsection. The basic physics is as follows: flux through a one-cycle forces the cycle to expand to lower the energy density contained in the flux. Similarly, flux through the dual to this cycle forces the dual cycle to expand. At fixed total volume, the combination of these two flux effects tends to stabilize the ratio of the sizes of the cycles and their duals. As we will see in $\S 2.1$, in order to achieve this stabilization for all the independent complex structure moduli, we will require $2 h$ independent fluxes threading the one-cycles of $\Sigma$. 


\subsection{Calculating the flux potential}

We would like to compute the potential energy obtained from the flux kinetic energy for one-form fields threading one-cycles on a Riemann surface. We do this explicitly for the cases of genus $h=1,2,3$, for which the period matrix (reviewed below) provides a faithful representation of the Riemann-surface complex-structure moduli space. This enables us to organize the problem as a simple change of basis between the integral basis appropriate to quantized fluxes and the holomorphic basis defining the period matrix. Our analysis may generalize to higher-genus examples if the extra directions in the period-matrix description can be dealt with.

Consider a genus $h$ Riemann surface $\Sigma$ with homology basis $a_{i}, b_{i}$ and integral oneforms $\alpha_{i}, \beta_{i}$ such that

$$
\begin{gathered}
\int_{a_{i}} \alpha_{j}=\delta_{i j} \\
\int_{b_{i}} \beta_{j}=\delta_{i j}
\end{gathered}
$$

and for any one-form $\eta$

$$
\begin{aligned}
\int_{a_{i}} \eta & =\int_{\Sigma} \eta \wedge \beta_{i} \\
\int_{b_{i}} \eta & =\int_{\Sigma} \alpha_{i} \wedge \eta .
\end{aligned}
$$

We wish to calculate the potential $U_{\text {flux }}=\int_{\Sigma} F \wedge * F$ for a one-form field $F$ in terms of the vector $\left(m_{i}, n_{i}\right)$ such that $F=m_{i} \alpha_{i}+n_{i} \beta_{i}$ (up to a shift by an exact 1 -form). This becomes cleaner by a transformation to a holomorphic basis where the Hodge star is easily defined. We can take a standard basis of $h$ holomorphic one-forms $\omega_{i}$ with antiholomorphic partners $\bar{\omega}_{i}$ such that

$$
\begin{gathered}
\int_{a_{i}} \omega_{j}=\delta_{i j} \\
\int_{b_{i}} \omega_{j}=\tau_{i j}
\end{gathered}
$$

for some symmetric period matrix $\tau$ with $\operatorname{Im} \tau$ positive definite.

Considering our flux one-form in the $\omega$ basis and in the $\alpha, \beta$ basis

$$
F=u_{i} \omega_{i}+\bar{u}_{i} \bar{\omega}_{i}=m_{i} \alpha_{i}+n_{i} \beta_{i}+\text { exact }
$$

we can see that

$$
\begin{gathered}
\int_{a_{i}} F=\delta_{i j} u_{j}+\delta_{i j} \bar{u}_{j}=m_{i} \\
\int_{b_{i}} F=\tau_{i j} u_{j}+\bar{\tau}_{i j} \bar{u}_{j}=n_{i}
\end{gathered}
$$


so we can define a $2 h \times 2 h$ matrix $K$ such that

$$
\begin{aligned}
K & =\left(\begin{array}{ll}
I & I \\
\tau & \bar{\tau}
\end{array}\right) \\
\left(\begin{array}{c}
m \\
n
\end{array}\right) & =K\left(\begin{array}{c}
u \\
\bar{u}
\end{array}\right) .
\end{aligned}
$$

Now the Hodge star acts on forms in the $\omega$ basis as

$$
\begin{aligned}
& * \omega=-i \omega \\
& * \bar{\omega}=i \bar{\omega}
\end{aligned}
$$

so it transforms the coefficients through the action of the matrix $H$ defined as

$$
H=\left(\begin{array}{cc}
-i I & 0 \\
0 & i I
\end{array}\right)
$$

Also, from (2.4) we know that $\int_{\Sigma} \alpha_{i} \wedge \beta_{j}=\delta_{i j}$, so altogether we get

$$
U_{\text {flux }}=\left(\begin{array}{ll}
m n & n
\end{array}\right) M K H K^{-1}\left(\begin{array}{c}
m \\
n
\end{array}\right)
$$

where

$$
M=\left(\begin{array}{cc}
0 & I \\
-I & 0
\end{array}\right) .
$$

To calculate everything in terms of $\tau$, we need to invert $K$. We find

$$
K^{-1}=\left(\begin{array}{cc}
-(\tau-\bar{\tau})^{-1} \bar{\tau} & (\tau-\bar{\tau})^{-1} \\
(\tau-\bar{\tau})^{-1} \tau & -(\tau-\bar{\tau})^{-1}
\end{array}\right)
$$

Multiplying, we get

$$
M K H K^{-1}=i\left(\begin{array}{cc}
2 \tau(\tau-\bar{\tau})^{-1} \bar{\tau} & -(\tau+\bar{\tau})(\tau-\bar{\tau})^{-1} \\
-(\tau-\bar{\tau})^{-1}(\tau+\bar{\tau}) & 2(\tau-\bar{\tau})^{-1}
\end{array}\right)
$$

For $h=1$ this reduces to

$$
M K H K^{-1}=\frac{2 i}{\tau-\bar{\tau}}\left(\begin{array}{cc}
|\tau|^{2} & -\operatorname{Re} \tau \\
-\operatorname{Re} \tau & 1
\end{array}\right) .
$$

So we obtain a flux potential energy

$$
U_{\text {flux }} \propto \sum_{I=1}^{N_{F}} Q^{i I} A_{i}^{j}(\tau) Q_{j}^{I}
$$


where $I$ indexes $N_{F}$ different types of flux with one component on $\Sigma, i$ and $j$ index the quantum numbers on the $a$ and $b$ cycles, and $A_{i}^{j}(\tau)$ is the $2 h \times 2 h$ matrix

$$
A(\tau)=i\left(\begin{array}{cc}
2 \tau(\tau-\bar{\tau})^{-1} \bar{\tau} & -(\tau+\bar{\tau})(\tau-\bar{\tau})^{-1} \\
-(\tau-\bar{\tau})^{-1}(\tau+\bar{\tau}) & 2(\tau-\bar{\tau})^{-1}
\end{array}\right)
$$

In (2.16) we have indicated the full dependence on $\tau$, while further dependence on volumes will arise in a complete construction via further compactification (and conversion to fourdimensional Einstein frame) in a way to be discussed explicitly below.

If we have higher-form fluxes that wrap cycles on more than one Riemann surface, the flux quantum numbers will just gain extra indices to be contracted by $A$, e.g. for a three form flux wrapping one-cycles of three different Riemann surfaces, the potential contribution is

$$
U_{\text {flux }} \propto Q^{i k m} A_{i}^{j}\left(\tau_{1}\right) A_{k}^{l}\left(\tau_{2}\right) A_{m}^{n}\left(\tau_{3}\right) Q_{j l n}
$$

where $\tau_{1}, \tau_{2}, \tau_{3}$ are the period matrices of the three surfaces and the flux indices can vary over different ranges if the genera of the Riemann surfaces are different.

\subsection{Analysis of $\tau$ stabilization}

We would like to understand if the potential energy (2.16) is sufficient to metastabilize the complex-structure moduli $\tau$. If we can show that the potential blows up at all the boundaries of the moduli space, then it must have a minimum in the interior. 5 These boundaries are known-they correspond to the imaginary part of $\tau$ becoming degenerate or $\tau$ factoring into smaller Riemann surfaces (e.g. $\tau_{12} \rightarrow 0$ in the $h=2$ case.) The first boundary can be dealt with fairly easily if there are $2 h$ independent flux vectors: Since $A$ is a positive-definite symmetric matrix, it has all positive eigenvalues. As an eigenvalue of $\tau-\bar{\tau}$ approaches $0,(\tau-\bar{\tau})^{-1}$ has an eigenvalue that gets large. This dominates the other components of $A$, meaning that $A$ must get a large eigenvalue, but with $2 h$ independent fluxes at least one has a component along the eigenvector with large eigenvalue, and thus the inner product (2.16) goes to infinity.

This argument does not deal with the factorization boundary, which is of a different character. The first type of boundary involves the shrinking of a nontrivial cycle, which

5 In fact, since the one-form fluxes spontaneously break modular invariance, at fixed flux quantum numbers we are interested in the boundary of the covering space of the Riemann-surface moduli space. 
some flux wraps, while the factorization limit involves the shrinking of a trivial cycle, which no flux wraps. However, for the case of small genus, we can explicitly find local minima of the flux potential for $\tau$ away from all boundaries. At $h=3$, a computer search of 100 linearly-independent flux choices found minima away from the boundary in all cases. It is possible to vary the flux choices, which allows some degree of tuning of the solutions for $\tau$. The computer search demonstrated a wide variation in the locations, which suggests that they are indeed tunable. It would be nice to have a conceptual argument for why the factorization boundary is avoided-a similar effect occurs in the case of the large complex structure limit of flux-stabilized Calabi-Yau compactifications [3].

\section{The volume and dilaton tadpoles: basic strategy}

Having fixed the complex-structure moduli via the flux potential described in the last section, we now turn to the stabilization of the dilaton and volume moduli. As discussed above, we have a tree-level tadpole for these quantities after compactifying on $\Sigma$. For our case of $h \geq 2$, this tree-level contribution to the potential energy is positive, driving the volume toward large values and the dilaton toward weak coupling. We need to introduce further ingredients in order to stabilize these directions, while also stabilizing additional runaway moduli introduced by the new ingredients, and doing it all in a way consistent with a controlled perturbative approximation scheme. This (in our experience) requires some trial and error, which results in the class of de Sitter models which we present in the next section. However, much of the input is based on simple intuition about the forces at play in compactifications with flux and branes [40], so we will start in this section by sharing our basic strategy.

Because all sources of Einstein-frame potential energy go to zero at weak coupling and large volume, stabilization requires sufficiently strong negative contributions. To obtain de Sitter space we aim for three terms in increasing orders of perturbation theory about weak coupling and large volume, with the middle term negative. For example, for the string coupling $g$, fixing the other moduli at their ultimate minima we require a potential of the form $a g^{2}-b g^{3}+c g^{4}$ which for large enough $b$ and $c$ produces a metastable minimum at weak coupling. In our examples, the tree-level contribution (2.2), and (2.16) in the case of NS 3-form flux, produces a positive term proportional to $g^{2}$. We can add a negative term at order $g^{3}$ (the order at which orientifolds and anti-orientifolds, the simplest such 
negative contribution, arise) and use the RR flux appearing at order $g^{4}$ to provide a final positive term.

In fact, we will now argue that there is a more general way to obtain a tunably large negative contribution generalizing that discussed in $\S 2.1$ of [3], by using the fact that wrapped intersecting branes and related curvature contributions can produce negative D3brane tension and charge.

Consider first the low-energy supergravity case of [3], starting from F-theory compactifications on elliptically fibered Calabi-Yau fourfolds. The base of the fibration has 7-branes at the loci where the $T^{2}$ fiber degenerates. There is an anomalous contribution to the D3-brane charge, given for a Calabi-Yau fourfold by $-\chi_{4} / 24$ where $\chi_{4}$ is the Euler character of the fourfold. There is correspondingly a supersymmetric partner of this charge, an effective 3 -brane tension also given by $-\chi_{4} / 24$. By varying the choice of fourfold, one can tune this contribution to large negative values. (In the IIB language this tunably-large anomalous charge and corresponding tension is associated in large part to inflow on intersections of 7-branes as in [42].) For perhaps the simplest case of a CY-fourfold fibered over $\mathbb{P}^{1} \times \mathbb{P}^{1} \times \mathbb{P}^{1}, \chi_{4} / 24=732$ 43, which yields a control parameter of order $10^{-3}$; more general fibrations can yield $\chi_{4} / 24$ significantly larger 44 .

In the Calabi-Yau case there is a positivity condition among a subset of contributions to the potential. 6 Specifically the NSNS and RR 3-form fluxes $H_{3}$ and $F_{3}$ are related to the anomalous 3-brane charge by Gauss's law:

$$
\frac{1}{(2 \pi)^{4}\left(\alpha^{\prime}\right)^{2}} \int_{\Sigma^{3}} H_{(3)} \wedge F_{(3)}=Q_{3,7}
$$

where $Q_{3,7}$ is the anomalous 3-brane charge of the 7-branes. In the low energy supersymmetric case, the anomalous charge is related by a BPS condition to the anomalous negative tension. This implies [3] that the combination of the $H_{3}$ and $F_{3}$ flux potential terms and the anomalous negative term is positive semidefinite.

But in the non-supersymmetric case there will generically be both 7-branes and anti7-branes. Adding extra sets of 7-branes and anti-7-branes to the base leads to triple intersections which locally each preserve $1 / 8$ of the ambient supersymmetry, and hence behave identically to the local contributions in a CY-fourfold; i.e. they contribute to the effective 3-brane tension. However, these pairs of branes will carry no net 3-brane

6 We thank R. Blumenhagen and F. Denef for reminding us of this important relation and the resulting need for the more generic ingredients to follow. 
charge, which will excuse us from the positivity relation that applies in the SUSY cased. Specifically, if we add extra sets of 7-branes and anti 7-branes, each in the same combination of 24 appearing in F-theory on K3, then the net negative contribution to the tension follows from adding these local sources and there will be no contribution to (3.1). More generally, we can also consider the possibility of extra $D 7-\overline{D 7}$ contributions, which will contribute to the same localized three-brane tension term.

Adding branes leads, a priori, to further brane moduli in need of stabilization, as in [3,1]. Although we add both branes and antibranes, there is a way to include them which minimizes their mutual interactions. Namely, in both the supersymmetric and non-supersymmetric cases, appropriate combinations of $6(\mathrm{p}, \mathrm{q})$ 7-branes do not carry net 7-brane charge (the combinations corresponding to $S O(8)$ points on K3). So such sets of 6 branes separated from sets of 6 antibranes avoid extra gauge attraction. As a result, these defects behave, from the point of view of each surface, as a set of massive sources in the effective $2+1$ dimensional gravity system. These effective masses get contributions from both the 7-brane and anti 7-brane tensions (included for $n_{7}$ (p,q) sets of 24 in (2.2)), and the anomalous negative tension we just reviewed which appears at the next order in $g_{s}$ perturbation theory. Note that because of the anomalous negative contribution, there is a large negative offset to their effective masses in the $2+1$ gravity problem on each surface. In particular, in the presence of both branes and antibranes, one might worry that they will tend to annihilate. However, with the negative contribution in place, the annihilation will not be energetically preferred in expansion about our solution. This is reminiscent of the fact that orientifolds and antiorientifolds do not annihilate (indeed a more prosaic, but less tunable, contribution at the same order would come from explicit $O 3-\overline{O 3}$ pairs).

So we will introduce such sets of branes and antibranes to obtain a tunably large negative tension contribution independent of the charge. But as in the original low-energy supersymmetric models [3,1] we will not explicitly stabilize the 7-brane moduli, leaving this to the genericity of the flux contributions and other corrections which can locally lift these non-chiral modes.8 Though we strongly expect generic contributions to indeed lift these non-chiral moduli, this will be the least explicit part of our construction.

So far we have only discussed Calabi-Yau F-theory models, with addional localized sources. Let us now return to our main interest, type IIB string theory in the critical

7 They will also break supersymmetry at the KK scale, just like the Riemann surfaces themselves.

8 See [45] for an explicit model in the low energy SUSY context eliminating the 7-brane moduli. 
dimension, compactified on a product of Riemann surfaces. Let us consider, for example, the above case of a CY fourfold fibered over $\mathbb{P}^{1} \times \mathbb{P}^{1} \times \mathbb{P}^{1}$, but sew in handles to the base $\mathbb{P}^{1}$ factors in smooth regions away from the 7 -branes, as well as adding extra sets of 7-branes and anti-7-branes as just discussed. For a general (non-Calabi-Yau) fourfold, the anomalous 3-brane charge is proportional to 46, 47]

$$
-\frac{1}{768}\left(\operatorname{tr}\left(R^{2}\right)\right)^{2}+\frac{1}{192} \operatorname{tr} R^{4}
$$

In the regions containing the handles, this contribution vanishes because the manifold is a product of the handle times a 3-manifold in that region and the traces in (3.2) vanish for this configuration (to be specific, one may consider adding handles and brane-antibrane pairs to the CY base in its orientifold limit 48,49,50]). As a result, the tunably large negative contribution to the 3-brane tension survives to a good approximation. It is only approximate because supersymmetry is broken, and the local relation between the anomalous 3-brane charge and tension is corrected. However, at large volume the supersymmetry is broken at a scale much lower than the scale of the 3-brane tension, so the BPS relation between the anomalous contribution to the charge and that of the effective 3-brane tension is still a good approximation as long as we ultimately stabilize the system in the large-volume regime.

Altogether, these ingredients yield a term in the effective potential of the form

$$
U_{3} \sim-\frac{1}{l_{4}^{4}} N_{7} \frac{g_{s}^{3}}{V^{2}}
$$

where $V$ is the total volume of the compactification, and $N_{7}$ is the effective control parameter just described coming from the triple intersections of 7 -branes. In particular $N_{7}$ has a contribution scaling like $\left(24 n_{7}\right)^{3} / 24$ in the notation of (2.2). More generally, there are other contributions. One could add $n_{D 7}$ sets of $D 7-\overline{D 7} \mathrm{~s}$, which would lead to contributions to $N_{7}$ scaling like cubic combinations of $n_{D 7}$ and $n_{7}$. One could also include explicit $O 3-\overline{O 3}$ pairs. In writing down (3.3) we have used the fact that the anomalous contributions do not depend on any geometric moduli in the string frame (and only depend on the overall volume in via the volume factor coming from the conversion to four dimensional Einstein frame).

In what follows we will include 7-branes via a term of the form (3.3), including also their positive 7-brane tension as in (2.2), and show how the volumes and dilaton are stabilized. We will not here explicitly determine where the 7-branes become localized 
inside the compactification, we expect ambient fluxes, dS thermal effects, and quantum corrections to generically lift these moduli. As discussed above, by combining the branes and antibranes each in appropriate groups of $(p, q)$ objects we can avoid strong forces between them.

\section{A new class of de Sitter models}

With the above inputs and motivations, we present our class of de Sitter models.

\subsection{The ingredients}

Consider type IIB string theory in the critical dimension compactified on a product of three Riemann surfaces $\Sigma_{s}$ of genera $h_{s}, s=1,2,3$. On each Riemann surface, we insert 7-branes and anti-7-branes yielding a net positive contribution to $N_{7}$, as defined above, with enough 7-brane-anti-7-brane pairs to obtain a tunably large net negative contribution to the part of the potential coming from the 3-form fluxes to be included and (3.3). Each 7-brane sits at a point in one of the $\Sigma_{s}$ factors and wraps a four cycle consisting of the other two Riemann surfaces. The control parameter $N_{7}$ behaves cubically in the 7-brane number, a feature that will figure into our analysis of the size of corrections. For simplicity, we will often take $h_{1}=h_{2}=h_{3}=h$ in the following.

In addition, we include the following types of flux in the compactification. First some notation: let $i_{s}=1, \ldots 2 h_{s}$ index the 1 -cycles on $\Sigma_{s}$. As above, let $s=1,2,3$ index the Riemann surface factors $\Sigma_{s}$. The ingredients are:

(1) Neveu-Schwarz 3-form flux $H_{3}$ on 3-cycles consisting of one one-cycle in each of the three $\Sigma$ factors. Let us denote these flux quantum numbers $N^{i_{1} i_{2} i_{3}}$.

(2) Ramond-Ramond 3-form flux $F_{3}$ on 3-cycles consisting of one one-cycle in each of the three $\Sigma$ factors. Let us denote these flux quantum numbers $Q_{3}^{i_{1} i_{2} i_{3}}$.

The contributions (1) and (2) must satisfy the constraint that $\int H_{3} \wedge F_{3}$ cancel the tadpole in three-brane charge arising from the net intersecting wrapped 7-branes described above (3.1).

(3) Ramond-Ramond 1-form flux on the 1-cycles of $\Sigma_{s}$; the corresponding flux quantum numbers will be denoted $Q_{1}^{s}$. This contribution will not play a significant role in our stabilization mechanism, but can be included among this class of models. 
(4) Ramond-Ramond 5-form flux on the 5-cycles consisting of 1-cycles of $\Sigma_{s}$ times $\Sigma_{s+1} \times$ $\Sigma_{s+2}$ where the subscripts are reduced mod 3. The corresponding flux quantum numbers will be denoted $Q_{5}^{I_{s}}$.

There is an interesting subtlety with this contribution. As discussed in [51], in an orientifold limit of the system of 24 -branes on $\mathbb{P}^{1}, T^{2} /\left(I_{2} \Omega(-1)^{F_{L}}\right)$, we cannot consider 5 -form flux with one index transverse to the O7-plane. That is, the zero mode of this flux is projected out by the orientifold action. More precisely, the flux must vanish at the positions of the O7-planes; all the KK excitations of this sort (with zeros at the O7-planes) are projected in and are hence consistent configurations.

Of course Kaluza-Klein excitations of the RR flux would not be suitable for our model building, since such modes necessarily fluctuate in time. Fortunately in our case we add handles to the $\mathbb{P}^{1}$, and the topology of the Riemann surfaces come to the rescue. The holomorphic 1-forms, which correspond to static solutions of the equations of motion for the flux, have zeros on higher genus Riemann surfaces (at genus $h$ the holomorphic 1-forms each have $2 h-2$ zeros by the Riemann-Roch theorem). Starting from a configuration where we sew handles onto the orientifold limit of the $\mathbb{P}^{1}$ with 247 -branes, in the $\Sigma_{i}$ direction in which our 5 -form flux reduces to a 1 -form we must consider the 1 -form with zeros at the positions of the orientifold planes. The flux solution is determined by the integer flux quanta and the complex structure only up to cohomology, so the zeros can be placed at the correct points by adding exact 1-forms. Having addressed this constraint in the orientifold limit, the setup should be consistent for more general configurations: nonsingular deformations of the compactification away from this limit will not change topological features such as the number of zeros. More generally, fluxes with boundary conditions that they must vanish at the positions of certain defects may be accommodated by considering sufficient genus to obtain enough zeroes in the corresponding holomorphic 1-forms. In general cases, this requires our flux stabilization in $\S 2$ to persist for higher genus.

\subsection{The low-energy spectrum}

In what follows we will analyze the potential for the potentially runaway moduli coming from the dilaton and the the volumes of the three Riemann surfaces. Because of the absence of isometries, we do not expect massless off diagonal components in the metric, both scalars that would modify the product structure of our base manifold and massless gauge fields in four dimensions arising from the $10 \mathrm{~d}$ metric. However there are other light 
degrees of freedom in the low-energy effective field theory arising from our construction. In particular, there are RR gauge fields and axions from dimensional reduction of the 10 dimensional RR potentials on the cycles of our compactification.

Let us consider the axions. These come from the type IIB RR scalar $C_{0}$ and harmonic 2 -forms made from the NS and RR potentials $B_{2}$ and $C_{2}$ integrated over 2-cycles of the compactification, as well as the RR 4-form $C_{4}$ on 4-cycles. The IIB axion $C_{0}$ couples via the term

$$
U_{C_{0}} \propto \int\left|F_{3}-C_{0} H_{3}\right|^{2}
$$

In general, one can solve $C_{0}$ 's equation of motion and plug the result into (4.1), yielding a more complicated potential energy for the other moduli (including $\tau$ ) than that arising in the absence of the $C_{0}$ coupling. This potential may generically have nontrivial local minima, but we can simplify the situation by arranging the fluxes to produce a solution at or near $C_{0}=0$. That is, we can arrange that the $C_{0}$ tadpole cancel by setting to zero the coefficient of its linear term in (4.1)

$$
U_{C_{0} \text { tadpole }} \propto C_{0} \int\left(H_{3} \wedge * F_{3}+F_{3} \wedge * H_{3}\right) \equiv 0 .
$$

This condition is consistent with our requirement to simultaneously turn on $\int H_{3} \wedge F_{3}$ to satisfy the Gauss' law constraint (3.1).

The axions arising from the 2-forms $B_{2}$ and $C_{2}$ have similar couplings:

$$
U_{C_{2}, B_{2}} \propto \int\left|F_{5}-\frac{1}{2} C_{2} \wedge H_{3}+\frac{1}{2} B_{2} \wedge F_{3}\right|^{2}
$$

We can also arrange a solution with negligible tadpoles for $C_{2}$ and $B_{2}$ by insisting that the coefficient of the linear term for these axions $\left(\int_{2-\text { cycles }} C_{2}\right.$ and $\left.\int_{2 \text {-cycles }} B_{2}\right)$ be small. The number of conditions on the flux choices this entails is $2 b_{2}$ where $b_{2}=12 h^{2}+3$ (given the same genus $h$ for all three Riemann surfaces). The number of 3 -form fluxes alone is $16 h^{3}$ so it is possible to accommodate these conditions, leaving behind enough independent fluxes to have $2 h$ independent fluxes threading one-cycles on each Riemann surface factor. Similar comments apply to the $C_{4}$ field, whose contribution scales like that of $C_{2}$.

If we made more general choices than those yielding $C_{0} \sim 0 \sim \int B_{2} \sim \int C_{2} \sim \int C_{4}$, we would obtain a somewhat more complicated effective potential for the other moduli. This may be interesting to study. In any case, having made these simplifications in the pseudoscalar sector, we move on to the potential energy for the volume moduli and the dilaton. 


\subsection{The potential energy}

We combine the sources described in the previous sections, including (2.2) (2.16) (3.3). We will denote the volume in string units of the $\Sigma_{s}$ factors $V_{s}$.

$$
\begin{aligned}
U= & \frac{1}{l_{4}^{4}} \frac{g_{s}^{4}}{\left(V_{1} V_{2} V_{3}\right)^{2}}\left\{\sum_{r} \frac{1}{g_{s}^{2}} 2\left(h_{r}+n_{7}-1\right) V_{r+1} V_{r+2}+\frac{1}{g_{s}^{2}} N^{i_{1} i_{2} i_{3}} A\left(\tau_{1}\right)_{i_{1}}^{j_{1}} A\left(\tau_{2}\right)_{i_{2}}^{j_{2}} A\left(\tau_{3}\right)_{i_{3}}^{j_{3}} N_{j_{1} j_{2} j_{3}}\right. \\
& -\frac{1}{g_{s}} N_{7}+Q_{3}^{i_{1} i_{2} i_{3}} A\left(\tau_{1}\right)_{i_{1}}^{j_{1}} A\left(\tau_{2}\right)_{i_{2}}^{j_{2}} A\left(\tau_{3}\right)_{i_{3}}^{j_{3}} Q_{3} j_{1} j_{2} j_{3} \\
& \left.+\sum_{r}\left[Q_{1}^{i_{r}} A\left(\tau_{r}\right)_{i_{r} j_{r}} Q_{1}^{j_{r}} V_{r+1} V_{r+2}+Q_{5}^{i_{r}} A\left(\tau_{r}\right)_{i_{r} j_{r}} Q_{5}^{j_{r}} \frac{1}{V_{r+1} V_{r+2}}\right]\right\}
\end{aligned}
$$

where we take the $r$ index to range cyclically over the labels $1,2,3$ of the three Riemann surface factors. Here the overall factor comes from the conversion to four-dimensional Einstein frame and the first term is from the Einstein-Hilbert action, dimensionally reduced as in (2.2). We have included the offset from the 7 -branes adding to the $2 h-2$ coefficient of (2.1). The second term contains the NS flux contribution, as in (2.18); the next is the effective 3-brane tension contribution from intersecting wrapped 7-branes, as in (3.3). The last terms give the RR flux contributions. We have dropped several order-one factors; we will keep track of the dependence on our discrete parameters which will provide parametric control.

\subsection{Metastable minima of the moduli potential}

In this subsection, we will elucidate how this model, with appropriately tuned choices of flux and brane quantum numbers, produces metastable de Sitter minima. We will first recall the result of $\S 1$ that (with sufficient independent fluxes) the complex moduli of each $\Sigma$ are metastabilized. Then we will observe that e.g. for an approximately symmetric distribution of fluxes, the relative volumes $V_{r} / V_{s}$ get stabilized at order 1 by the potential (4.4). Finally, we will demonstrate the stabilization of the overall volume and dilaton.

These manipulations will lead us to tuning requirements on the flux and brane quantum numbers, as well as expressions for how the stabilized values of the moduli scale with these discrete quantum numbers. In the following subsection we will use these results to make standard estimates for the size of the $\alpha^{\prime}$ and $g_{s}$ corrections to the background, which must be tuned small self-consistently. We will see that the required tuning is available in our system given the possibility of scaling up the contribution $N_{7}$ as discussed above.

Complex moduli of $\Sigma$ 
In $\S 1$, we established that the complex-structure moduli $\tau$ of the Riemann surface are stabilized by $2 h$ independent fluxes threading one-cycles of $\Sigma$. The 1 -form and 5 -form fluxes alone are sufficient to stabilize the complex moduli of a genus 2 Riemann surface. More generally, the 3 -form fluxes lead to $4 h_{r+1} h_{r+2}$ types of fluxes on $\Sigma_{r}$, allowing one to stabilize the complex structure moduli of products of higher genus surfaces as well.

\section{Ratios of $\Sigma$ volumes}

In (4.4), the positive terms in the potential are symmetric among the three $\Sigma$ factors. Let us scale out the dependence on the overall volume $V=V_{1} V_{2} V_{3}$ in each term. The positive terms in the resulting potential all go to infinity for any large ratio of volumes $V_{r} / V_{s}$. If we tune the fluxes to be approximately symmetric among the three $\Sigma_{r}$ factors, then we obtain a minimum at $V_{r} / V_{s} \sim 1$ from these terms 9 .

\section{Overall volume and dilaton}

Let us now move to the problem of stabilizing the overall volume $V=V_{1} V_{2} V_{3}$ and dilaton. Setting the relative volumes $V_{r} / V_{s} \equiv 1$ our potential reduces to one of the form

$$
\begin{aligned}
U\left(g_{s}, V\right) & =C\left(g_{s}^{2}\left(h+n_{7}-1\right) \frac{1}{V^{4 / 3}}+g_{s}^{2} n_{3}^{2} \frac{1}{V^{2}}\right. \\
& -g_{s}^{3} N_{7} \frac{1}{V^{2}} \\
& \left.+g_{s}^{4} q_{3}^{2} \frac{1}{V^{2}}+g_{s}^{4} q_{1}^{2} \frac{1}{V^{4 / 3}}+g_{s}^{4} q_{5}^{2} \frac{1}{V^{8 / 3}}\right)
\end{aligned}
$$

Here for simplicity we have taken the fluxes to be symmetrically distributed among the three $\Sigma$ factors, each of genus $h$. We have introduced the shorthand $n_{3}^{2}$ for the flux potential from the NS 3-form, evaluated at the minimum $\tau=\tau_{*}$, and similarly for the Ramond fluxes $q_{a}^{2}$

Consider first the first, third, and sixth terms in (4.5). These form a fourth degree polynomial in the combination $g_{s} / V^{2 / 3}$ so for appropriate choices of coefficients, they stabilize this combination at

$$
\frac{g_{s}}{V^{2 / 3}} \sim \frac{h+n_{7}-1}{N_{7}} \sim \frac{N_{7}}{q_{5}^{2}}
$$

9 More generally, one can use the symmetry of the potential and the Arithmetic MeanGeometric Mean inequality to show that the volumes are stabilized at values of order the ratios of flux numbers. 
Plugging this into the remaining terms (the second, fourth, and fifth) in (4.5), we obtain a contribution

$$
U_{2-4-5} \sim\left(\frac{h+n_{7}-1}{N_{7}}\right)^{2} n_{3}^{2} \frac{1}{V^{2 / 3}}+\left(\frac{h+n_{7}-1}{N_{7}}\right)^{4} q_{3}^{2} V^{2 / 3}+\left(\frac{h+n_{7}-1}{N_{7}}\right)^{4} q_{1}^{2} V^{4 / 3}
$$

If we consider flux choices such that the first two terms here dominate (while remaining subdominant to the first, third and sixth terms discussed above), the second set of terms will not destabilize $g_{s} / V^{2 / 3}$ and we find $V$ stabilized such that

$$
V^{4 / 3} \sim\left(\frac{N_{7} n_{3}}{\left(h+n_{7}-1\right) q_{3}}\right)^{2}
$$

while the string coupling is stabilized at

$$
g_{s} \sim \frac{n_{3}}{q_{3}}
$$

This procedure for stabilizing the volume and dilaton requires that the terms (4.7) are subdominant to the first, third and sixth terms in (4.5). In particular, the 3-form NS and RR flux potentials at the minimum, $n_{3}^{2}$ and $q_{3}^{2}$, need to be sufficiently small so that the corresponding terms are subdominant. As discussed above, at the same time, we must satisfy (3.1), which ties the flux quantum numbers $N_{3}$ and $Q_{3}$ to net 7-brane contributions to the effective D3-charge. Given the combinations of branes and antibranes in our construction, which contribute to the anomalous negative tension but not the charge, this is not a problem.

\section{The cosmological constant and moduli masses}

The set of metastable minima we have exhibited produces a discretuum of possible cosmological constants, depending on the flux choices. If we do not tune coefficients significantly to obtain a low cosmological constant, then the scale of the resulting cosmological constant is of order the Kaluza-Klein scale of the compactification

$$
\Lambda_{\text {untuned }} \sim \frac{h}{V^{1 / 3}} \frac{1}{\alpha^{\prime}}
$$

By tuning our discrete parameters we can arrange the cosmological constant and moduli masses to produce a hierarchy of scales between the KK scale of the compactification and the curvature scale in four dimensions as well as the moduli mass scales:

$$
\Lambda_{\text {tuned }}, m_{\text {moduli }}^{2} \ll \Lambda_{\text {untuned }}
$$




\section{Anti de Sitter examples}

Although we have focused on de Sitter minima, similar methods lead to anti-de Sitter vacua. For example, in situations where the negative 7-brane contribution is sufficiently strong relative to the RR flux contributions, our minimum may dip below zero.

To obtain de Sitter solutions, a necessary condition is at least three independent contributions in each direction in an expansion about weak coupling/large volume, with the middle term negative. More generally, to obtain parts of the discretuum that are purely anti-de Sitter, we can consider other compactifications which only provide two terms (negative, positive) in some or all the directions. This allows one to consider products of Riemann surfaces with spheres or orbifolds of spheres.

\subsection{Estimates of subleading corrections and self-consistency checks}

In the above analysis, we exhibited metastable minima with small string coupling and large volumes (relative to the string scale). Although this is a necessary condition for a controlled solution, there are further self-consistency checks we must make. In the presence of large flux and brane quantum numbers, it is necessary to check that the effective expansion parameters coming into stringy and quantum corrections are small. These expansion parameters are somewhat enhanced by the large discrete quantum numbers. Hence in this

subsection, we will systematically estimate the size of the corrections taking these factors into account.

\section{Curvature corrections}

String theory has a generic expansion in $\alpha^{\prime} \mathcal{R}$ where $\mathcal{R}$ is the curvature of the spacetime background. By tuning (4.8) large, we can preclude these large curvature corrections in our type IIB background. Specifically, in string units,

$$
\mathcal{R} \sim \frac{h}{V^{1 / 3}} \sim \frac{h\left(h+n_{7}-1\right)^{1 / 2}}{N_{7}^{1 / 2}}\left(\frac{q_{3}}{n_{3}}\right)^{1 / 2} \ll 1 .
$$

Keeping in mind that $q_{3} / n_{3} \sim 1 / g_{s}$, this requires that $N_{7}$ be large.

Note that as in [3, [1], we are using a large number of 7-branes to obtain a strong negative contribution in the potential energy. In F-theory (or related M-theory or IIA backgrounds) this is itself related to an $\mathcal{R}^{4}$ correction. It would be nice to check explicitly whether there are any other enhanced quartic curvature contributions, and if so if these are independently tunable. This question also arises in the low energy supersymmetric models, and we believe it is reasonable to take the approach of [3] 目, and assume that 
these other $\mathcal{R}^{4}$ contributions are subdominant since the large number introduced by the intersecting branes contributing to $\chi_{4}$ need not generally contribute coherently to other curvature terms. Indeed, this statement was checked for some situations in [52]. Note, as discussed above, that adding handles to the base in itself adds a small correction to the $\chi_{4} \mathcal{R}^{4}$ contribution.

NS flux corrections

Next let us consider the expansion in $\left(H_{3} \alpha^{\prime}\right)^{2} \sim n_{3}^{2} / V$. To make this small we require

$$
\left(\frac{\left(h+n_{7}-1\right)}{N_{7}}\right)^{3 / 2} n_{3}^{1 / 2} q_{3}^{3 / 2} \ll 1
$$

which is weaker than (4.12) since we have already insisted that $n_{3} q_{3} \ll N_{7}$.

$R R$ flux corrections

The RR flux vertex operators come with an additional factor of $g_{s}$. Hence the condition for control of the higher derivative terms involving a $p$-form flux is $g_{s}^{2} q_{p}^{2} / V^{p / 3} \ll 1$. For the threeform flux $F_{3}$, this is satisfied in a similar way to that described above for $H_{3}(4.13)$.

For the 5-form RR flux we obtain the condition

$$
\frac{N_{7} g_{s}}{V} \sim \frac{\left(h+n_{7}-1\right)^{3 / 2}}{N_{7}^{1 / 2}}\left(\frac{q_{3}}{n_{3}}\right)^{1 / 2} \ll 1
$$

which is the strongest condition we encounter. Since $N_{7} \sim 24^{2} n_{7}^{3}$, this contribution is of order $g_{s}^{-1 / 2} 10^{-3 / 2}$. This permits reasonable control if we consider also a $g_{s}$ of order $1 / 10$. If one includes additional contributions coming from $D 7-\overline{D 7}$ pairs in such a way that they contribute positively to $N_{7}$, this control becomes parameteric.

\section{Quantum corrections}

Finally, we need to consider the expected strength of quantum corrections to our Lagrangian in this background. At high energy-momentum flowing through the loops, above the KK scale, the contributions are localized in the compactification and the effective coupling is the $10 \mathrm{~d}$ coupling $g_{*} \sim n_{3} / q_{3}$ (4.9). We therefore choose $q_{3}$ at least somewhat larger than $n_{3}$. At low energies, in the $4 \mathrm{~d}$ effective field theory, the corrections scale like $g^{2} / V$ times the number of species running in loops. This number is roughly $N_{7}$ at low energies (if we keep the genus $h$ low enough that topological enhancements from the handles are subdominant). Putting this together with the above scalings, we have

$$
\frac{N_{7} g_{s}^{2}}{V} \sim \frac{\left(h+n_{7}-1\right)^{3 / 2}}{N_{7}^{1 / 2}}\left(\frac{n_{3}}{q_{3}}\right)^{1 / 2} \ll 1 .
$$

This is weaker than (4.14) by a factor of $g_{s}$. 


\section{Discussion}

This class of models provides a perturbative set of de Sitter vacua to a good approximation, as long as the non-chiral 7-brane moduli are lifted as expected by the ambient fluxes and quantum corrections (an issue common to this case and the low energy supersymmetric models). In particular, we exhibited sufficient controlled forces to stabilize the string coupling and volumes, as well as the Riemann surface complex structure moduli, perturbatively. It further illustrates the fact that the string theory "landscape" goes beyond the low energy supergravity sectors most studied to date (though these models may still permit low-energy supersymmetry in the matter sector).) In particular, in studying the discretuum of string vacua, it does not suffice to consider only those with a low-energy supergravity effective Lagrangian in four dimensions.

\subsection{Number of Vacua}

As anticipated in [4], like other examples of flux vacua our construction leads to the possibility of mass production of metastable string vacua, by varying over the many possible flux, brane, and topological quantum numbers. Let us estimate roughly the number of vacua in our new class of models. As discussed in [4], a rough estimate for the number of vacua is obtained by counting the volume in a sphere in flux space up to a maximum radius determined by the strength of the "bare" negative cosmological constant to be cancelled by the fluxes. In our case, as in [1] the "bare" negative piece is dominated by the 7-brane contribution in (4.4). This is cancelled in part by flux squared contributions, so we can roughly trade this for $M^{2}$ where $M$ scales like the dominant flux quantum numbers. Let us denote the maximal value of this quantity (determined by the maximal value of $N_{7}$ available including back reaction constraints) by $M_{\text {max }}$. Our space of fluxes is $2 b_{3}+b_{1}+b_{5}$ dimensional (where $b_{p}$ are the numbers of noncontractible $p$-cycles in the compactification). Putting this together, our estimate for the number of vacua available here is

$$
N_{v a c} \sim\left(\frac{M_{\max }^{2}}{b_{3}+\left(b_{1}+b_{5}\right) / 2}\right)^{b_{3}+\left(b_{1}+b_{5}\right) / 2}
$$

Note that relative to the Calabi-Yau case, the extra handles enhance the dimension of the flux space. In general, one might expect that relaxing conditions such as the Calabi-Yau condition enhances the number of independent ingredients, hence increasing the number of vacua in the more generic starting points. In (5.1) we see one aspect of that here. Of 
course the examples we have studied themselves constitute only a small corner of the space of compactifications.

It would be interesting to determine the distributions of these vacua in moduli space, as in [10]. It is clear that the assumption of low energy supersymmetric effective Lagrangians must be relaxed in order to obtain a representative sample of string vacua, and to answer related questions about the statistics of the supersymmetry-breaking scale [田, 2, [33]. This point, already clear from the case of string scale supersymmetry breaking, is reinforced by the intermediate class of models discussed here.

\subsection{Holographic Duality}

The new de Sitter construction may also be of interest for studying the microphysics of de Sitter space, since the perturbative ingredients involved in the moduli stabilization, including the volume stabilization, are fairly explicit. As before, the fluxes can be traded for branes to expose some of the microphysical content of the holographic duals on their approximate moduli space [53]. In this regard it is interesting that as in the construction [1], here we required nontrivial NS flux $\mathrm{H}_{3}$, which leads to the presence of NS-branes in the approximate moduli space of the dual. It would be interesting to know if this is part of a general pattern.

\subsection{String Duality}

These compactifications raise interesting questions concerning string-string duality. One possible way to explore this would be to elucidate more explicitly whether there is a useful $\mathrm{F}$ theoretic description of $T^{2}$ fibrations over more generic base manifolds (such as the $\Sigma^{3}$ in the present construction).

Another possibility is to try to understand the small-radius behavior of compactifications on Riemann surfaces. In the present work, we tuned to obtain a set of large-radius compactifications in order to maintain general relativity as a good approximation. However, the small-radius limit of the Riemann surface may remain a well-defined conformal field theory if we take into account strong worldsheet dynamics (and remain on shell by including the time dependence arising from the tadpoles, or by including other ingredients to metastabilize the space). It is even possible that the theory grows dimensions at strong worldsheet coupling; this possibility is perhaps suggested by the fact that the form of the tree-level dilaton tadpole in our theory (2.1)

$$
U_{\mathcal{R}} \sim \frac{1}{l_{4}^{4}}(2 h-2) \frac{g_{s}^{2}}{V_{\Sigma}^{2} V_{X}}
$$


is reminiscent of that in dimension $D$ supercritical string theory models [54] [2]

$$
U_{D} \sim \frac{1}{l_{4}^{4}}(D-10) \frac{g_{s}^{2}}{V_{\text {internal }}}
$$

In both these formulas there is an integer quantum number in the coefficient-which serves as the effective central charge which must be soaked up by dilaton time dependence or higher order balancing of forces. It is related to the first Chern class of the manifold in the case of Riemann-surface compactifications, and is related to the dimensionality in the case of supercritical limits of string theory. It would be very interesting to understand if these two integers are related to each other by a duality, for example in the small radius regime of the Riemann surface compactifications.

\subsection{Phenomenological applications}

Finally, it would be interesting to explore the phenomenology of these models. One question to ask is whether low-energy SUSY in the particle physics sector may emerge in some models of the sort we consider here (cf. for example [38]). In particular, gravitational communication of the SUSY breaking of the gravity sector to the Standard Model leads to superpartner mass squares of order $m_{K K}^{4} / M_{p}^{2}$. This alone would provide $T e V$ scale SUSY breaking in the observable sector if $m_{K K} \sim 10^{11} \mathrm{GeV}$, though there may be other mediation mechanisms which dominate this depending on the details. In any case, more generically, we expect many models with high-scale SUSY breaking in this context; it would be interesting to check for models of the sort [7]. String-theoretic standard-model constructions based on intersecting branes on tori might fit into this framework well, as locally there are products of circles in our $\Sigma^{3}$ compactifications. 10

\section{Acknowledgements}

We thank P. Aspinwall, R. Blumenhagen, F. Denef, S. Dimopoulos, M. Dine, L. Fidkowski, J. Hsu, A. Kashani-Poor, X. Liu, L. McAllister, J. McGreevy, A. Pierce, M. Schulz, C. Vafa, and especially S. Kachru and A. Tomasiello for many useful discussions. We thank the organizers and participants of the String Vacuum Workshop in Munich, and the SLAC Theory group, for useful comments when this work was first presented. We are supported in part by the DOE under contract DE-AC03-76SF00515 and by the NSF under contract 9870115 .

10 We thank M. Schulz for this comment. 


\section{References}

[1] S. Kachru, R. Kallosh, A. Linde and S. P. Trivedi, "De Sitter vacua in string theory," Phys. Rev. D 68,046005 (2003) arXiv:hep-th/0301240].

[2] A. Maloney, E. Silverstein and A. Strominger, "De Sitter space in noncritical string theory," arXiv:hep-th/0205316; E. Silverstein, "(A)dS backgrounds from asymmetric orientifolds," arXiv:hep-th/0106209.

[3] S. B. Giddings, S. Kachru and J. Polchinski, "Hierarchies from fluxes in string compactifications," Phys. Rev. D 66, 106006 (2002) arXiv:hep-th/0105097.

[4] R. Bousso and J. Polchinski, "Quantization of four-form fluxes and dynamical neutralization of the cosmological constant," JHEP 0006, 006 (2000) arXiv:hep-th/0004134.

[5] C. S. Chan, P. L. Paul and H. Verlinde, "A note on warped string compactification," Nucl. Phys. B 581, 156 (2000) arXiv:hep-th/0003236.

[6] B. S. Acharya, "A moduli fixing mechanism in M theory," arXiv:hep-th/0212294.

[7] N. Arkani-Hamed and S. Dimopoulos, "Supersymmetric unification without low energy supersymmetry and signatures for fine-tuning at the LHC," arXiv:hepth/0405159; G. F. Giudice and A. Romanino, "Split supersymmetry," Nucl. Phys. B 699, 65 (2004) arXiv:hep-ph/0406088.

[8] M. Becker, G. Curio and A. Krause, "De Sitter vacua from heterotic M-theory," Nucl. Phys. B 693, 223 (2004) [arXiv:hep-th/0403027.

[9] A. Saltman and E. Silverstein, "The scaling of the no-scale potential and de Sitter model building," arXiv:hep-th/0402135.

[10] S. Ashok and M. R. Douglas, "Counting flux vacua," JHEP 0401, 060 (2004) arXiv:hep-th/0307049. F. Denef and M. R. Douglas, "Distributions of flux vacua," JHEP 0405, 072 (2004) arXiv:hep-th/0404116. A. Giryavets, S. Kachru and P. K. Tripathy, "On the taxonomy of flux vacua," arXiv:hep-th/0404243; O. de Wolfe, A. Giryavets, S. Kachru, W. Taylor, "Enumerating enhanced symmetries in flux vacua", arXiv:hep-th/0411061.

[11] S. Gukov, C. Vafa and E. Witten, "CFTs from Calabi-Yau Fourfolds," Nucl. Phys. B584, 69 (2000) arXiv:hep-th/9906070

[12] L. Susskind, "The anthropic landscape of string theory," arXiv:hep-th/0302219.

[13] C. P. Burgess, R. Kallosh and F. Quevedo, "de Sitter string vacua from supersymmetric D-terms," JHEP 0310, 056 (2003) arXiv:hep-th/0309187.

[14] S. Kachru, M. B. Schulz and S. Trivedi, "Moduli stabilization from fluxes in a simple IIB orientifold," JHEP 0310, 007 (2003) arXiv:hep-th/0201028.

[15] A. R. Frey and J. Polchinski, "N = 3 warped compactifications," Phys. Rev. D 65, 126009 (2002) arXiv:hep-th/0201029.

[16] P. K. Tripathy and S. P. Trivedi, "Compactification with flux on K3 and tori," JHEP 0303, 028 (2003) arXiv:hep-th/0301139. 
[17] K. Dasgupta, G. Rajesh and S. Sethi, "M theory, orientifolds and G-flux," JHEP 9908, 023 (1999) arXiv:hep-th/9908088.

[18] T. R. Taylor and C. Vafa, "RR flux on Calabi-Yau and partial supersymmetry breaking," Phys. Lett. B 474, 130 (2000) arXiv:hep-th/9912152.

[19] P. Mayr, "On supersymmetry breaking in string theory and its realization in brane worlds," Nucl. Phys. B 593, 99 (2001) arXiv:hep-th/0003198.

[20] L. Andrianopoli, S. Ferrara and M. Trigiante, "Fluxes, supersymmetry breaking and gauged supergravity," arXiv:hep-th/0307139.

[21] F. Marchesano, G. Shiu and L. T. Wang, "Model building and phenomenology of flux-induced supersymmetry breaking on D3-branes," arXiv:hep-th/0411080.

[22] J. P. Conlon and F. Quevedo, "On the explicit construction and statistics of CalabiYau flux vacua," JHEP 0410, 039 (2004) arXiv:hep-th/0409215.

[23] V. Balasubramanian and P. Berglund, "Stringy corrections to Kaehler potentials, SUSY breaking, and the cosmological constant problem," arXiv:hep-th/0408054.

[24] P. G. Camara, L. E. Ibanez and A. M. Uranga, "Flux-induced SUSY-breaking soft terms on D7-D3 brane systems," arXiv:hep-th/0408036; D. Lust, S. Reffert and S. Stieberger, "Flux-induced soft supersymmetry breaking in chiral type IIb orientifolds with D3/D7-branes," arXiv:hep-th/0406092.

[25] R. Blumenhagen, D. Lust and T. R. Taylor, "Moduli stabilization in chiral type IIB orientifold models with fluxes," Nucl. Phys. B 663, 319 (2003) arXiv:hep-th/0303016.

[26] L. Gorlich, S. Kachru, P. K. Tripathy and S. P. Trivedi, "Gaugino condensation and nonperturbative superpotentials in flux compactifications," arXiv:hep-th/0407130.

[27] J. J. Blanco-Pillado et al., "Racetrack inflation," arXiv:hep-th/0406230.

[28] H. Firouzjahi, S. Sarangi and S. H. H. Tye, "Spontaneous creation of inflationary universes and the cosmic landscape," JHEP 0409, 060 (2004) arXiv:hep-th/0406107.

[29] E. I. Buchbinder, "Raising anti de Sitter vacua to de Sitter vacua in heterotic Mtheory," arXiv:hep-th/0406101.

[30] M. B. Schulz, "Superstring orientifolds with torsion: O5 orientifolds of torus fibrations and their massless spectra," Fortsch. Phys. 52, 963 (2004) arXiv:hep-th/0406001.

[31] S. B. Giddings and R. C. Myers, "Spontaneous decompactification," Phys. Rev. D 70, 046005 (2004) arXiv:hep-th/0404220.

[32] M. Berg, M. Haack and B. Kors, "Loop corrections to volume moduli and inflation in string theory," arXiv:hep-th/0404087.

[33] T. Banks, M. Dine and E. Gorbatov, "Is there a string theory landscape?," arXiv:hep-th/0309170; M. Dine, E. Gorbatov and S. Thomas, arXiv:hep-th/0407043;

M. R. Douglas, "Statistical analysis of the supersymmetry breaking scale," arXiv:hepth/0405279. L. Susskind, "Supersymmetry breaking in the anthropic landscape," arXiv:hep-th/0405189; E. Silverstein, "Counter-intuition and Scalar Masses", arXiv:hepth/0407202. 
[34] G. Dvali, "Large hierarchies from attractor vacua," arXiv:hep-th/0410286.

[35] P. K. Townsend and M. N. R. Wohlfarth, "Accelerating cosmologies from compactification," Phys. Rev. Lett. 91, 061302 (2003) arXiv:hep-th/0303097.

[36] N. Ohta, "Accelerating Cosmologies and Inflation from M/Superstring Theories," arXiv:hep-th/0411230.

[37] M. Bershadsky, A. Johansen, V. Sadov and C. Vafa, "Topological reduction of 4-d SYM to 2-d sigma models," Nucl. Phys. B 448, 166 (1995) arXiv:hep-th/9501096.

[38] M. A. Luty, "Weak scale supersymmetry without weak scale supergravity," Phys. Rev. Lett. 89, 141801 (2002) arXiv:hep-th/0205077.

[39] R. Kallosh and A. Linde, "Landscape, the scale of SUSY breaking, and inflation," arXiv:hep-th/0411011.

[40] E. Silverstein, "TASI / PiTP / ISS lectures on moduli and microphysics," arXiv:hepth/0405068; to appear in the proceedings of TASI 2003.

[41] C. Vafa, "Evidence for F-Theory," Nucl. Phys. B 469, 403 (1996) arXiv:hepth/9602022]. ; D. R. Morrison and C. Vafa, "Compactifications of F-Theory on CalabiYau Threefolds - I," Nucl. Phys. B 473, 74 (1996) [arXiv:hep-th/9602114. D. R. Morrison and C. Vafa, "Compactifications of F-Theory on Calabi-Yau Threefolds - II," Nucl. Phys. B 476, 437 (1996) [arXiv:hep-th/9603161]. M. Bershadsky, K. A. Intriligator, S. Kachru, D. R. Morrison, V. Sadov and C. Vafa, "Geometric singularities and enhanced gauge symmetries," Nucl. Phys. B 481, 215 (1996) arXiv:hep-th/9605200

[42] Y. K. Cheung and Z. Yin, "Anomalies, branes, and currents," Nucl. Phys. B 517, 69 (1998) arXiv:hep-th/9710206. R. Minasian and G. W. Moore, "K-theory and Ramond-Ramond charge," JHEP 9711, 002 (1997) arXiv:hep-th/9710230.

[43] A. Klemm, B. Lian, S. S. Roan and S. T. Yau, "Calabi-Yau fourfolds for M- and F-theory compactifications," Nucl. Phys. B 518, 515 (1998) arXiv:hep-th/9701023.

[44] http://hep.itp.tuwien.ac.at/ kreuzer/CY

[45] F. Denef, M. Douglas, B. Florea, and S. Kachru, in progress.

[46] S. Sethi, C. Vafa and E. Witten, "Constraints on low-dimensional string compactifications," Nucl. Phys. B 480, 213 (1996) arXiv:hep-th/9606122.

[47] K. Becker and M. Becker, "M-Theory on Eight-Manifolds," Nucl. Phys. B 477, 155 (1996) arXiv:hep-th/9605053.

[48] A. Sen, "Orientifold limit of F-theory vacua," Nucl. Phys. Proc. Suppl. 68, 92 (1998) [Nucl. Phys. Proc. Suppl. 67, 81 (1998)] arXiv:hep-th/9709159.

[49] R. Gopakumar and S. Mukhi, "Orbifold and orientifold compactifications of F-theory and M-theory to six and four dimensions," Nucl. Phys. B 479, 260 (1996) arXiv:hepth/9607057].

[50] K. Dasgupta, G. Rajesh and S. Sethi, "M theory, orientifolds and G-flux," JHEP 9908, 023 (1999) arXiv:hep-th/9908088. 
[51] C. Angelantonj, S. Ferrara and M. Trigiante, "New D = 4 gauged supergravities from $\mathrm{N}=4$ orientifolds with fluxes," JHEP 0310, 015 (2003) [arXiv:hep-th/0306185].

[52] K. Becker, M. Becker, M. Haack and J. Louis, "Supersymmetry breaking and alpha'-corrections to flux induced potentials," JHEP 0206, 060 (2002) arXiv:hepth/0204254.

[53] M. Fabinger and E. Silverstein, "D-Sitter space: Causal structure, thermodynamics, and entropy," arXiv:hep-th/0304220; A. Karch, "Auto-localization in de-Sitter space," JHEP 0307, 050 (2003) arXiv:hep-th/0305192]; E. Silverstein, "AdS and dS entropy from string junctions," arXiv:hep-th/0308175; M. Alishahiha, A. Karch, E. Silverstein and D. Tong, "The dS/dS correspondence," arXiv:hep-th/0407125.

[54] R. C. Myers, "New Dimensions For Old Strings," Phys. Lett. B 199, 371 (1987). S. P. de Alwis, J. Polchinski and R. Schimmrigk, "Heterotic Strings With Tree Level Cosmological Constant," Phys. Lett. B 218, 449 (1989). 